Dedicated to Murray Gerstenhaber and Jim Stasheff

\title{
TOPICS IN ALGEBRAIC DEFORMATION THEORY
}

\author{
ANTHONY GIAQUINTO
}

\begin{abstract}
We give a selective survey of topics in algebraic deformation theory ranging from its inception to current times. Throughout, the numerous contributions of Murray Gerstenhaber are emphasized, especially the common themes of cohomology, infinitesimal methods, and explicit global deformation formulas.
\end{abstract}

\section{INTRODUCTION}

The concept of deformation is pervasive in mathematics. Its aim is to study objects of some type by organizing them into continuous families and determine how objects in the same family are related. This is the classic problem of determining the "moduli" of an algebra or of a more general structure. The moduli are, roughly, the parameters on which the structure depends. The idea goes back at least to Riemann who, in his famous treatise [Ri57] on abelian functions, showed that the Riemann surfaces of genus $g$ form a single continuous family of dimension $3 g-3$. This family is the prototype of a moduli space, a concept central to deformation theory.

The modern era of deformations began with the pioneering work of FröhlicherNijenhuis FN57 and Kodaira-Spencer KS58 on deformations of complex manifolds. In particular, we see in FN57 the first formal use of infinitesimal (cohomological) methods in a deformation problem as the authors prove that if $X$ is a complex manifold, $T$ its sheaf of holomorphic tangent vectors, then there can be no perturbation of the complex structure whenever $H^{1}(X, T)=0$. In the monumental treatise KS58, Kodaira and Spencer then developed a systematic theory of deformations of complex manifolds, including the infinitesimal and obstruction theories. For the case of Riemann surfaces, there are no obstructions as $H^{2}(X, T)=0$.

Algebraic deformation theory began with Gerstenhaber's seminal paper Ge64. Although the analytic theory served as a model, numerous new concepts lie within the realm of algebraic deformation theory. In fact, all formal aspects of analytic deformations of manifolds are special cases of those in the algebraic theory - this will be made precise in section 8 .

Infinitesimal methods for algebra deformations are governed by Hochschild cohomology. The study of infinitesimals led to the discovery of the Gerstenhaber algebra structure on $H H^{*}(A, A)$, see [Ge63]. The ingredients of a Gerstenhaber algebra - compatible graded Lie and commutative products - occupy a central position in "higher structures in mathematics and physics." Another key higher structure is that of the various infinity algebras: $A_{\infty}, L_{\infty}$ and their generalizations. These structures have roots in Stasheff's landmark treatise Sta63, which, coincidentally appeared in the same year as Ge63. While disjoint at the time, the ideas in Gerstenhaber's and Stasheff's 1963 papers would become closely intertwined in the years to come. Indeed, Gerstenhaber called the entire Hochschild cohomology $H H^{*}(A, A)$ the "infinitesimal ring" of $A$ in Ge63, even though only 
the components of $H H^{i}(A, A)$ with $i \leq 3$ had natural interpretations related to infinitesimals and obstructions. But more than 30 years later, it became well known that the entire Hochschild cohomology $H^{*}(A, A)$ is the space of infinitesimals of deformations of $A$ to an $A_{\infty}$ algebra.

This survey only represents a sampling of ideas in algebraic deformation theory. None of the discussion is new, except for the results in the last section on algebra variations. Many ideas are only sketched and proofs are omitted. More topics are left out than included. In particular, the theory of deformation quantization is largely left out - the reader is referred to Sternheimer's contribution [Ste] in this volume and the references therein for the important physical perspective. Also left out is the deformation theory of infinity algebras, gerbes, stacks, chiral algebras, affine and dynamical quantum groups, and the like. More comprehensive surveys of algebraic deformation theory and quantization can be found in the excellent treatises [GS88] CKTB05] and [DMZ07.

\section{Algebraic Deformations}

Let $A$ be an associative algebra over a commutative ring $k$.

Definition 1. A formal deformation of $A$ is a $k[[t]]$-algebra $A_{t}$ which is flat and $t$-adically complete as a $k[[t]]$-module, together with an isomorphism $A \simeq A_{t} / t A_{t}$.

For every deformation, there is a $k[[t]]$-module isomorphism between $A_{t}$ and $A[[t]]$. Once such an isomorphism is fixed, the multiplication in $A_{t}$ is necessarily of the form

$$
\mu_{t}: A[[t]] \otimes_{k[[t]]} A[[t]] \rightarrow A[[t]] \quad \text { with } \quad \mu_{t}(a, b)=a b+\mu_{1}(a, b) t+\mu_{2}(a, b) t^{2}+\cdots
$$

where $a b$ represents the multiplication of $A$ and each $\mu_{i} \in \operatorname{Hom}(A \otimes A, A)$ is extended to be $k[[t]]$-bilinear. Setting $\mu_{0}(a, b)=a b$, we have $\mu_{t}=\sum \mu_{i} t^{i}$ and $\mu_{t}(a, b)$ will be denoted $=a * b$.

It is clear that one can consider formal deformations of other algebraic structures (Lie algebras, bialgebras, algebra homomorphisms, etc. ) by modifying the above definition to suit the appropriate category. The deformation of an algebraic structure is usually subjected to the same equational constraints as the original structure:

- Associative algebra $A:(a * b) * c=a *(b * c)$. If $A$ is commutative we can also require $a * b=b * a$.

- Lie algebra $L:[a, b]_{t}=-[b, a]_{t}$ and the Jacobi identity for $[a, b]_{t}=[a, b]+$ $[a, b]_{1} t+[a, b]_{2} t^{2}+\cdots$.

- Bialgebra $B$ : associativity of $*$, coassociativity of $\Delta_{t}(a)=\Delta(a)+\Delta_{1}(a) t+$ $\cdots$, and $\Delta_{t}(a * b)=\Delta_{t}(a) * \Delta_{t}(b)$.

- Algebra homomorphism $\phi: A \rightarrow A^{\prime}: \phi_{t}(a b)=\phi_{t}(a) \phi_{t}(b)$ with $\phi_{t}(a)=$ $\phi(a)+\phi_{1}(a) t+\phi_{2}(a) t^{2}+\cdots$.

Even though in this note we are concerned with formal deformations, there are many important and explicit instances for which the deformed products converge or are even polynomial in $t$ when $k=\mathbb{R}$ or $\mathbb{C}$.

\section{The Deformation philosophy of Gerstenhaber}

The pioneering principle of Gerstenhaber is that the equational constraints above can be naturally interpreted in terms of the appropriate cohomology groups and 
higher structures on them. In particular, the infinitesimal (linear term of the deformation) is a cocycle in the cohomology group - Hochschild $H H^{2}(A, A)$ in the associative case, Harrison $\operatorname{Har}^{2}(A, A)$ in the commutative case, Chevalley-Eilenberg $H_{C E}^{2}(L, L)$ in the Lie case, Gerstenhaber-Schack $H_{G S}^{2}(B, B)$ in the bialgebra case, and the diagram cohomology $H_{d}^{2}(\phi, \phi)$ in the algebra homomorphism case. Moreover, the obstructions to extending infinitesimal and $n$-th order deformations to global ones are controlled by the differential graded Lie algebra structure on the cohomology.

In the associative case, the graded Lie structure (and much more) was laid out in Ge63. There it was shown that the Hochschild cohomology $H H^{*}(A, A)=$ $\bigoplus_{n \geq 0} H^{n}(A, A)$ has a remarkably rich structure consisting of two products,

- A graded commutative product where $\operatorname{deg} H H^{p}(A, A)=p$,

- A graded Lie product where $\operatorname{deg} H H^{p}(A, A)=p-1$,

- $[\alpha,-]$ is a graded derivation of the commutative product.

A graded $k$-module satisfying the above conditions is a Gerstenhaber algebra. Other notable examples are $\bigwedge^{*} L$ (where $L$ is a Lie algebra), $H^{*}\left(X, \bigwedge^{*} T\right.$ ) (where $X$ is a manifold and $T$ is its sheaf of tangent vectors), and the diagram cohomology $H_{d}^{*}(\mathbb{A}, \mathbb{A})$ of an arbitrary presheaf $\mathbb{A}$ of $k$-algebras (to be defined in Section 8 ). The Chevalley-Eilenberg, Harrison, and bialgebra cohomology cohomologies carry graded Lie brackets, but are not Gerstenhaber algebras in general.

In Ge63, the commutative and Lie products on the Hochschild cohomology $H H^{*}(A, A)$ are defined at the cochain level and are proved to descend to the level of cohomology. An intrinsic interpretation of the graded Lie structure was given by Stasheff in Sta93. There he proved that the Gerstenhaber bracket coincides with the natural graded bracket on $\operatorname{Coder}(B A, B A)$, where $B A$ is the bar complex of $A$.

Returning to the equational constraints for a deformation of an algebra $A$, the associativity of $\mu_{t}$ can succinctly be expressed in terms of the Gerstenhaber bracket as $\left[\mu_{t}, \mu_{t}\right]=0$. Writing $\mu_{t}=\mu_{0}+\mu^{\prime}$ it follows that $2\left[\mu_{0}, \mu^{\prime}\right]+\left[\mu^{\prime}, \mu^{\prime}\right]=0$. Since the coboundary in the shifted Hochschild complex $C^{*}(A, A)[1]$ is $\delta=\left[\mu_{0},-\right]$, the first summand is $2 \delta \mu^{\prime}$. Thus we arrive at the fundamental associativity equivalences

$$
\mu_{t} \quad \text { associative } \Longleftrightarrow\left[\mu_{t}, \mu_{t}\right]=0 \quad \Longleftrightarrow \delta\left(\mu^{\prime}\right)+\frac{1}{2}\left[\mu^{\prime}, \mu^{\prime}\right]=0 .
$$

Thus $\mu_{t}$ is associative if and only if $\mu^{\prime}$ satisfies the Maurer-Cartan equation. Although not explicitly stated as such, the idea that deformations are governed by a differential graded Lie algebra and solutions to the Maurer-Cartan equation goes back to Gerstenhaber's original paper Ge64.

\section{Algebras with Deformations}

The search for deformations of an algebraic structure $A$ begins with the appropriate cohomology group (usually $H^{2}(A, A)$ ) which comprises the infinitesimals. Given an infinitesimal $\mu_{1}$, the basic question is whether it can be integrated to a full deformation or not. In other words, is it possible to find $\mu_{2}, \mu_{3}, \ldots$ such that $\mu^{\prime}=\sum_{i \geq 1} \mu_{i} t^{i}$ satisfies the Maurer-Cartan equation. Of course, the vanishing of the obstruction group (usually $H^{3}(A, A)$ ) guarantees that any infinitesimal is integrable, but this is rarely the case. A necessary condition in general is that the primary obstruction, $\left[\mu_{1}, \mu_{1}\right]$, must equal zero. There are then higher obstructions which must vanish in order for $\mu_{1}$ to be integrable. Thus, a reasonable starting 
point for deformations is to first determine which infinitesimals have a vanishing primary obstruction. Remarkably, in several fundamentally important cases, all infinitesimals $\mu_{1}$ with $\left[\mu_{1}, \mu_{1}\right]=0$ can be integrated. In fact, some of the most celebrated theorems in deformation theory are expressions of this phenomenon.

Perhaps the most studied algebra deformations are those which lie in the realm of deformation quantization, a concept introduced in the seminal paper BFFLS78. Suppose $X$ is a real manifold and $A=C^{\infty}(X)$. Then $H H^{2}(A, A)$ can be identified with the space of bivector fields $\alpha \in \Gamma\left(X, \wedge^{2} T\right)$, and the primary obstruction to $\alpha$ is the Schouten bracket $[\alpha, \alpha]$. The condition $[\alpha, \alpha]=0$ asserts that $\alpha$ determines Poisson structure on $X$. In BFFLS78 it was asked whether any Poisson structure can be quantized. The affirmative answer to this question is one of the jewels of deformation theory.

Theorem 1 (Kontsevich). Any Poisson manifold can be quantized. More generally, there is, up to equivalence, a canonical correspondence between associative deformations of the algebra $A$ and formal Poisson structures $\alpha_{t}=\alpha_{1} t+\alpha_{2} t^{2}+\cdots$ on $A$.

Also in Ko97 is a remarkable explicit quantization formula for $X=\mathbb{R}^{n}$. The formula involves certain weighted graphs which determine the $*$-product expansion. A physical interpretation of the deformation quantization formula in terms of path integrals of models in string theory was made precise by Cattaneo and Felder in CF00. In the case where $X$ is a smooth algebraic variety, quantization of Poisson brackets is also possible, but significant modifications of the approach are necessary, see Ko01, VdB07, and Ye05.

Another case where the primary obstruction to integrating an infinitesimal is the only one is in the realm of quantum groups. Consider a Lie bialgebra $\mathfrak{a}$. The cocommutator, $\delta: \mathfrak{a} \rightarrow \mathfrak{a} \otimes \mathfrak{a}$, can be extended to an infinitesimal deformation of the coalgebra structure of $U \mathfrak{a}$ which is compatible with the Lie bracket on $\mathfrak{a}$, and hence the multiplication in $U \mathfrak{a}$. The cocommutator may thus be viewed as an infinitesimal whose primary obstruction vanishes. Drinfel'd asked in [Dr92] whether any Lie bialgebra can be quantized. The affirmative answer to this question is another famous result in deformation theory.

Theorem 2 (Etingof-Kazhdan). Any Lie bialgebra can be quantized. That is, if $\mathfrak{a}$ is a Lie bialgebra, then there exists a Hopf algebra deformation of $U \mathfrak{a}$ whose infinitesimal is the cocommutator of $\mathfrak{a}$.

The quantization of $U \mathfrak{a}$ depends on a choice of Drinfel'd associator. It is known that associators are not unique and are notoriously difficult to compute with.

Many of the results pertaining to quantization of solutions to the various types of classical Yang-Baxter equation can also be viewed as examples of the phenomenon that in certain situations, the primary obstruction to integrating an infinitesimal structure is the only one. Some of these instances will be discussed in Section 7

In general, the condition $\left[\mu_{1}, \mu_{1}\right]=0$ does not guarantee that an infinitesimal $\mu_{1}$ is integrable. The earliest known example is geometric in nature and predates the algebraic theory. In [Do60, Douady exhibited an example of an infinitesimal deformation (in the Kodaira-Spencer sense) of the Heisenberg group whose primary obstruction vanishes, yet its secondary obstruction, a Lie-Massey bracket fails to vanish. More recently and in the algebraic case, Mathieu has given examples of commutative Poisson algebras which cannot be quantized, see Mat97. 


\section{Algebras without Deformations}

A deformation $A_{t}$ of an algebra $A$ is trivial if there is a $k[[t]]$-algebra isomorphism $\Phi_{t}: A_{t} \rightarrow A[[t]]$ which reduces the identity modulo $t$. An algebra is rigid if it has no non-trivial deformations. The cohomology results of Section 2 provide the first elementary result in deformation theory.

Theorem 3. If $H^{2}(A, A)=0$ then $A$ is rigid.

Algebras which satisfy the hypothesis of Theorem 3 are called absolutely rigid. Here are some notable examples of absolutely rigid algebras in various categories.

- Any separable $k$-algebra $A$ is rigid as these are characterized by $H H^{n}(A,-)$ for all $n \geq 1$.

- The enveloping algebra $U \mathfrak{g}$ of a finite dimensional semisimple Lie algebra $\mathfrak{g}$ is rigid as an algebra as $H H^{n}(U \mathfrak{g}, U \mathfrak{g})=0$ for $n \geq 1$. It does admit deformations as a Hopf algebra.

- The coordinate ring $\mathcal{O}(V)$ of a smooth affine variety $V$ is rigid as a commutative algebra as $\operatorname{Har}^{n}(\mathcal{O}(V), \mathcal{O}(V))=0$ for $n \geq 1$ - a more precise interpretation of the cohomology $\mathcal{O}(V)$ will be given in Section 6. It does admit noncommutative deformations however.

- The $m$-th Weyl (Heisenberg) algebra $A_{m}$ is rigid as $H H^{n}\left(A_{m}, A_{m}\right)=0$ for $n \geq 1$.

The converse of Theorem 3 is known to be false in many instances. Richardson has provided in Ri67] examples of rigid Lie algebras $L$ with $H_{C E}^{2}(L, L) \neq 0$. In the associative case, Gerstenhaber and Schack have given examples of rigid associative algebras when $\operatorname{char}(k)=p$ in [GS86]. Remarkably, the question of whether there exist rigid associative algebras $A$ in characteristic zero with $H H^{2}(A, A) \neq 0$ is still an open question even in the case where $A$ is a finite-dimensional $\mathbb{C}$-algebra.

The rigid algebras of Gerstenhaber and Schack in $\operatorname{char}(k)=p$ are not everyday examples. The smallest rigid algebra constructed with $H H^{2}(A, A) \neq 0$ is a 669dimensional algebra over $\mathbb{F}_{2}$. The algebra is a poset algebra of a suspension of a triangulation of the projective plane.

The proof of the rigidity of these algebras despite non-zero $H H^{2}(A, A)$ is based on an elementary but fundamental theorem of [GS86] concerning relative Hochschild cohomology. If $S$ is a subalgebra of $A$ then a cochain $F \in C^{n}(A, A)$ is $S$-relative if

$$
\begin{aligned}
F\left(s a_{1}, \ldots, a_{n}\right)=s F\left(a_{1}, \ldots, a_{n}\right), \quad F\left(a_{1}, \ldots, a_{n} s\right) & =F\left(a_{1}, \ldots, a_{n}\right) s, \\
& \text { and } F\left(\ldots, a_{i} s, a_{i+1}, \ldots\right)=F\left(\ldots, a_{i}, s a_{i+1}, \ldots\right)
\end{aligned}
$$

for all $s \in S$ and $a_{i} \in A$. Further, an $S$-relative cochain $F$ is reduced if $F\left(a_{1}, \ldots, a_{n}\right)=$ 0 whenever any $a_{i} \in S$.

Theorem 4. If $S$ is a separable subalgebra of $k$, then the inclusion of the complex of reduced $S$-relative cochains into the full Hochschild complex induces an isomorphism of cohomology.

The theorem significantly reduces cohomology computations whenever $A$ has a large separable subalgebra. For example, a poset algebra is a subset of the algebra of $n \times n$ matrices and one may take $S$ to be the diagonal matrices. Using Theorem 4 it is elementary to show that the Hochschild cohomology of the poset algebra coincides with the simplicial cohomology of the geometric realization or nerve (see Section 8) of the poset. 
Another application of the theorem is in the computation of the cohomology of a crossed product algebras. Let $V$ be a finite dimensional $k$-vector space, $S V$ its symmetric algebra, and $G$ a finite group which acts on $V$, and hence on $S V$. For $x \in S V$ and $g \in G$ denote the action of $g$ on $x$ by $x^{g}$. The crossed product $S V \ltimes G$ has underlying space $S V \otimes k G$, with the usual multiplication in $S V$ and $k G$, and relations $(1 \otimes g)(x \otimes 1)=x^{g} \otimes g$ for $x \in S V$ and $g \in G$. For simplicity, we omit the tensor product symbol and write an element $x \otimes g$ simply as $x g$.

When $|G|$ is invertible in $k$, then Maschke's Theorem asserts that $k G$ is separable - let us assume this here. In this case, we may compute $H H^{*}(S V \ltimes G, S V \ltimes G)$ using reduced $k G$-relative cochains. For such a cochain $F$, we have

$$
F\left(x_{1} g_{1}, x_{2} g_{2} \ldots, x_{n} g_{n}\right)=F\left(x_{1}, x_{2}^{g_{1}} \ldots, x_{n}^{g_{1} \cdots g_{n-1}}\right) g_{1} \cdots g_{n} .
$$

The right side of the equation is an element of $C^{n}(S V, S V \ltimes k G)$ and it is easy to see that it is $G$-invariant in the sense that

$$
g F\left(x_{1}, \ldots, x_{n}\right) g^{-1}=F\left(g x_{1} g^{-1}, \ldots, g x_{n} g^{-1}\right) .
$$

Therefore we obtain

$$
H H^{*}(S V \ltimes G, S V \ltimes G) \simeq\left(H H^{*}(S V, S V \ltimes G)\right)^{G} .
$$

A complete computation of the cohomology can be found in many sources, and one which explicitly uses $k G$-relative cohomology is [Pi06. There is interest in crossed product cohomology and deformations as they have geometric implications for orbifolds (see CGW04]) and in the theory of symplectic reflection algebras (see EG02 and Section 9 of this survey).

It is interesting to note that some of the rigid algebras listed above naturally appear in parametrized families, a seeming contradiction to the general theory. For example, consider $\mathcal{O}_{\lambda}=\mathbb{C}[x, y]$ with $y^{2}=x(x-1)(x-\lambda)$, the ring of regular functions on an affine elliptic curve. If $\lambda$ is close to $\lambda^{\prime}$ then $\mathcal{O}_{\lambda} ¥ \mathcal{O}_{\lambda^{\prime}}$. Nevertheless, $H H^{2}\left(\mathcal{O}_{\lambda}, \mathcal{O}_{\lambda}\right)=0$. According to Kontsevich, the problem here is that the variety, being affine, is not compact and formal deformation theory for non-compact objects can give "nonsensical" results, see Ko01. In the associative case, a "compact" object is a finite-dimensional algebra and so we expect other nonsensical results for some infinite-dimensional algebras. Here is such an example: the first quantized Weyl algebra $A_{q}=\mathbb{C}\langle x, y\rangle /(q x y-y x-1)$ is not isomorphic to $A_{1}$ for $q$ near 1 . However, as noted above, $H H^{2}\left(A_{1}, A_{1}\right)=0$. To put this into a formal deformation theoretic perspective, let $q=1+t$. Then there is indeed an analytic isomorphism $\phi: A_{1}[[t]] \rightarrow A_{q}[[t]]$, but it has zero radius of convergence. A similar phenomenon happens for the situation with $O_{\lambda}$ and $O_{\lambda^{\prime}}$. The problem is that passing to the formal power series versions of these algebras has trivialized the deformations.

The above examples suggest that the classic deformation theory of a single algebra does not always detect the dependency of an algebra on parameters. However, the more general diagram cohomology theory of Section 8 can detect such dependencies, but does not show how the algebras vary with the parameters. The construction of the algebras with varying moduli can be accomplished through the idea of a variation of algebras. This concept will be addressed in Section 10.

\section{Universal Deformation Formulas}

The process of constructing deformations using the infinite step-by-step procedure of extending deformations of order $n$ to $n+1$ for each $n \geq 1$ is impractical. 
There are instances though in which a closed form for $\mu_{t}$ is known. One is the explicit quantization of Poisson brackets on $\mathbb{R}^{n}$ given in [Ko97]. Another comes from the use of "universal deformation formulas" which are, in essence, Drinfel'd twists which act on a certain classes of algebras. The prototypical example of this type of formula was given by Gerstenhaber in Ge68. There it was observed that if $\phi$ and $\psi$ are commuting derivations of any associative algebra (in characteristic zero), then $a * b=\sum \phi^{n}(a) \psi^{n}(b) \frac{t^{n}}{n !}$ is associative. The most famous use of this idea gives the Moyal product. For example, if $A=k[x, y]$ with $\phi=\partial_{x}$ and $\psi=\partial_{y}$, then we have $x * y-y * x=t$, and the deformation is isomorphic to the first Weyl algebra as long as $t \neq 0$. When $\phi=x \partial_{x}$ and $\psi=y \partial y$ then the deformation is graded and isomorphic to the skew-polynomial $\operatorname{ring} k\langle x, y\rangle /(q x y-y x)$ with $q=e^{t}$. These examples can of course be extended to higher dimensions.

Definition 2. Suppose $B=(B, \Delta, 1, \epsilon)$ is a bialgebra with comultiplication $\Delta$, unit 1 , and counit $\epsilon$. A universal deformation formula (UDF) based on $B$ is an element $F \in(B \otimes B)[[t]]$ such that

$((\Delta \otimes 1)(F))(F \otimes 1)=((1 \otimes \Delta)(F))(1 \otimes F) \quad$ and $\quad(\epsilon \otimes 1) F=(1 \otimes \epsilon) F=1 \otimes 1$.

The virtue of a UDF is that for any $B$-module algebra $A$, the product $a * b=$ $\mu \circ F(a \otimes b)$ is associative and hence is a deformation of $A$, see GZ98.

Example 1. Suppose $B$ is commutative and let $r \in P \otimes P$ where $P$ is the space of primitive elements. Then $F=\exp (t r)$ is a UDF. Primitive elements of $B$ act as derivations of any B-module algebra and so this UDF gives a wide range of Moyal-type deformations.

Example 2. Let $B=U \mathfrak{s}$, where $\mathfrak{s}$ is the Lie algebra with basis $\{H, E\}$ and relation $[H, E]=E$. Set $H^{\langle n\rangle}=H(H+1) \cdots(H+n-1)$. Then $F=\sum \frac{t^{n}}{n !} H^{\langle n\rangle} \otimes E^{n}$ is UDF. For an example of its use, take $A=k[x, y]$ with the derivations $H=x \partial_{x}$ and $E=x \partial_{y}$. The deformed algebra has the relation $x * y-y * x=t x^{2}$ and is the Jordan quantum plane. Numerous generations of this UDF can be found in KLO01, LS02] and the references therein.

Example 3. Let $\mathfrak{g} \otimes \mathfrak{g}$ be a Lie algebra and let $r \in \mathfrak{g} \wedge \mathfrak{g}$ satisfy $[r, r]=0$, where $[-,-]$ is the Schouten bracket. Drinfel'd has shown in Dr83. that there exists a UDF $F=1 \otimes 1+t r+O\left(t^{2}\right)$. Examples 1 and 2 are of this form. It should be noted that $[r, r]=0$ means that $r$ is a skew-symmetric solution of the classical Yang-Baxter equation.

Example 4. Let $B$ be the bialgebra generated by $\left\{D_{1}, D_{2}, \sigma\right\}$ with relations $D_{1} D_{2}=$ $D_{2} D_{1}, \quad D_{i} \sigma=q \sigma D_{i}(i=1,2)$, and comultiplication

$$
\Delta\left(D_{1}\right)=D_{1} \otimes \sigma+1 \otimes D_{1}, \quad \Delta\left(D_{2}\right)=D_{2} \otimes 1+\sigma \otimes D_{1}, \quad \Delta(\sigma)=\sigma \otimes \sigma .
$$

Then $F=\exp _{q}\left(t D_{1} \otimes D_{2}\right)$ is a UDF, where the $q$-exponential is the usual exponential series with $n$ ! replaced by $n_{q}$ !.

Note that for any B-module algebra, $\sigma$ acts as an automorphism and. the elements $D_{1}, D_{2}$ act as commuting skew derivations with respect to $\sigma$. Thus, this UDF provides q-Moyal type deformations. For example, it can be used to deform the quantum plane $k\langle x, y\rangle /(q x y-y x)$ to the first quantized Weyl algebra 
$A_{q}=k\langle x, y\rangle /(q x y-y x-1)$. Formulas of this type were also used in CGW04] to deform certain crossed products $S V \ltimes G$.

Recently, universal deformation formulas have arisen naturally in the work of Connes and Moscovici on Rankin-Cohen brackets and the Hopf algebra $H_{1}$ of transverse geometry, see [CM04]. Rankin-Cohen brackets are families of bi-differential operators on modular forms. These brackets can be assembled to give universal deformation formulas. Some applications appear in CM04. The formulas based on $H_{1}$ are also connected to certain topics in deformation quantization as it relates to the Poisson geometry of groupoids and foliations. see [BTY07].

\section{Commutative Algebras and the Hodge Decomposition}

Let $A$ be a commutative algebra over a field of characteristic zero. In Ba68, Barr proved that the Harrison cohomology $\operatorname{Har}^{n}(A, A)$ is a direct summand of the Hochschild cohomology $H H^{n}(A, A)$. The key to this splitting was Barr's discovery of an idempotent $e_{n}$ in $\mathbb{Q} S_{n}$, the rational group algebra of the symmetric group. The symmetric group acts on $C^{n}(A, M)$ (the Hochschild $n$-cochains of $A$ with coefficients in a symmetric $A$-bimodule $M)$ via $\sigma F\left(a_{1}, \ldots, a_{n}\right)=F\left(a_{\sigma 1}, \ldots, a_{\sigma n}\right)$. Barr proved that $\delta\left(e_{n} F\right)=e_{n+1}(\delta F)$, where $\delta$ is the Hochschild coboundary operator. Thus $H H^{n}(A, M)$ splits as $e_{n} H H^{n}(A, M) \oplus\left(1-e_{n}\right) H H^{n}(A, M)$, and the latter piece is $\operatorname{Har}^{n}(A, M)$. Barr's work received little attention until 1987 when Gerstenhaber and Schack extended the splitting, see GS87. In $\mathbb{Q} S_{n}$ there are $n$ mutually orthogonal idempotents $e_{n}(1), \ldots, e_{n}(n)$ with the property that $\delta\left(e_{n}(r) F\right)=e_{n+1}(r)(\delta F)$ for all $F \in C^{n}(A, M)$. The relation between the idempotents and coboundary give the following fundamental theorem.

Theorem 5 (Hodge Decomposition). Suppose char $(k)=0$ and let $A$ be a commutative algebra and $M$ a symmetric A-bimodule. Then there is a splitting

$$
H H^{n}(A, M)=H H^{1, n-1}(A, M) \oplus H H^{2, n-2}(A, M) \oplus \cdots \oplus H H^{n, 0}(A, M)
$$

where $H H^{r, n-r}(A, M)$ is the cohomology of the complex $e_{*}(r) C^{*}(A, M)$.

Around the same time as the Gerstenhaber-Schack paper GS87, Loday, using different techniques, exhibited a splitting of the Hochschild and cyclic cohomologies of a commutative algebra, see [Lo88, Lo89].

The idempotents $e_{n}(r)$, which have independent interest apart from cohomology, are most easily described using the following elegant generating function discovered by Garsia in [Ga90]:

$$
\sum e_{n}^{(r)} x^{r}=\frac{1}{n !} \sum_{\sigma \in S_{n}} \operatorname{sgn}(\sigma)\left(x-d_{\sigma}\right)\left(x-d_{\sigma}+1\right) \cdots\left(x-d_{\sigma}+n-1\right) \sigma
$$

where $d_{\sigma}$ is the number of descents in $\sigma$, i.e. the number of $i$ with $\sigma(i)>\sigma(i+1)$.

The following diagram, in which $H H^{i, n-i}(A, M)$ is abbreviated as $H^{i, n-i}$, is instructive in understanding the Hodge decomposition. 


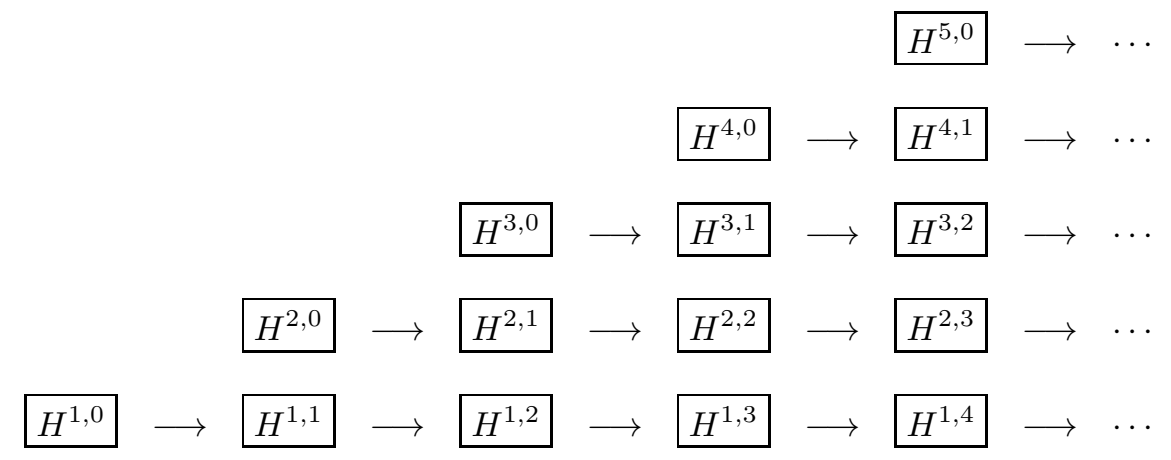

In the diagram, vertical columns represent the breakup of $H H^{n}(A, M)$, starting with $n=1$, and the horizontal arrows display the Hochschild coboundary. The bottom row, $H^{1, *}(A, M)$, is the Harrison cohomology $\operatorname{Har}(A, M)$ which is associated to Barr's idempotent. The idempotent $e_{n}(0)$ is the skew-symmetrizer $\frac{1}{n !} \sum_{\sigma \in S_{n}}(-1)^{\sigma} \sigma$ and it follows that the diagonal components, $H H^{n, 0}(A, M)$ are the skew multi-derivations, $\bigwedge_{A}^{n} \operatorname{Der}(A)$, of $A$ into $M$. If $A=\mathcal{O}(V)$, the ring of regular functions on a smooth affine variety $V$, then the celebrated Hochschild-KostantRosenberg Theorem asserts that $H H^{n}(A, A)=\bigwedge_{A}^{n} \operatorname{Der}(A)$, where $\operatorname{Der}(A)$. In terms of the Hodge decomposition, the Theorem becomes $H H^{n}(A, A)=H H^{n, 0}(A, A)$. In particular, $\operatorname{Har}^{2}(A, A)=0$ and these algebras have no commutative deformations. In the case $V$ is not smooth, one expects the components $H H^{r, n-r}(A, A)$ to encode information regarding the singularities. Some interesting results by Fronsdal in this direction can be found in [Fr07.

It is clear that the refinement of $H H^{*}(A, A)$ provided by the Hodge decomposition can be useful. For example, if $H H^{*}(A, A)$ is infinite dimensional, then its Euler-Poincare characteristic is not well-defined. However, its partial EulerPoincare characteristics (alternating sums of $\operatorname{dim} H^{r, *-r}(A, A)$ ) may all be defined. Here is an example which illustrates this phenomenon. Let $A=k[\epsilon] / \epsilon^{2}$ be the ring of dual numbers. It is well-known that $H^{n}(A, A)$ has dimension one for all $n \geq 1$. Using the Hodge decomposition, one can show that $H H^{n}(A, A)=H^{k, n-k}(A, A)$, where $k=\left\lfloor\frac{n+1}{2}\right\rfloor$. The partial Euler-Poincare characteristics are deformation invariant and as such they can be helpful in detecting whether a given scheme is a deformation of another one.

N. Bergeron and Wolfgang showed that the components $\bigoplus_{r=1}^{k} H H^{r, n-r}(A, A)$ consist of those classes of cocycles vanishing on $(k+1)$-shuffles but not on $m$-shuffles for any $m<k+1$, see BW95 for the precise definition and explanation. This generalizes the fact that Harrison cohomology consists of those cocycles vanishing on 2-shuffles. Another fact proved in BW95 is that $H H^{r, n-r}(A, A)$ behaves well with respect to the filtration $\mathcal{F}_{m}=\bigoplus_{r \geq m} H H^{*, r}(A, A)$ in the sense that $\left[\mathcal{F}_{p}, \mathcal{F}_{q}\right] \subset$ $\mathcal{F}_{p+q}$. 
Other instances of cohomology decompositions arising from group actions are possible. For example, F. Bergeron and N. Bergeron found in BB92 a type $B$ decomposition. Specifically, they showed that there are $n$ idempotents in the descent algebra of the Weyl group of type $B$, the group of signed permutations on $n$ letters. Moreover, if $A$ is an algebra with involution and $M$ is a symmetric $A$-bimodule, then there is an action of $B_{n}$ on $A^{\otimes n}$ with the property that the idempotents are compatible with the Hochschild coboundary map. Thus there is a "type $B$ " splitting of the cohomology. This raises the question of whether there are idempotents in the descent algebras of other Coxeter systems $(W, S)$ which decompose $H H^{*}(A, M)$ for algebras $A$ with a suitable $W$-action.

\section{Bialgebra Deformations}

It was clear that, after discovery of quantum groups in the 1980's, there should be a cohomology theory of bialgebras with the usual features related to deformations. In GS90a Gerstenhaber and Schack introduced such a theory which we now describe.

The Gerstenhaber-Schack bialgebra cohomology $H_{G S}^{*}\left(B, B^{\prime}\right)$ is defined for certain matched pairs of bialgebras $B$ and $B^{\prime}$. For simplicity, we only describe here the case $B^{\prime}=B$ (any bialgebra is matched with itself). Since $B$ is a bialgebra, any tensor power $B^{\otimes m}$ is both a $B$-bimodule and a $B$-bicomodule and thus the Hochschild cohomology $H H^{*}\left(B, B^{\otimes m}\right)$ and the coalgebra (Cartier) cohomology $H_{c}^{*}\left(B^{\otimes m}, B\right)$ are well-defined. Set $C^{p, q}(B, B)=\operatorname{Hom}_{k}\left(B^{\otimes p}, B^{\otimes q}\right)$. The Hochschild coboundary operator provides a map $\delta_{h}: C^{p, q}(B, B) \rightarrow C^{p+1, q}(B, B)$ while the coalgebra coboundary yields $\delta_{c}: C^{p, q}(B, B) \rightarrow C^{p, q+1}(B, B)$. These coboundaries commute giving the Gerstenhaber-Schack complex

$$
C_{G S}^{*, *}(B, B) \quad \text { with } \quad C_{G S}^{n}(B, B)=\bigoplus_{\substack{p+q=n \\ p, q>0}} C^{p, q}(B, B) \quad \text { and } \quad \delta_{G S}=\delta_{h}+(-1)^{q} \delta_{c} .
$$

The bialgebra cohomology $H_{G S}^{*}(B, B)$ is then the homology of this complex.

There are variants of this theory. For example, if one takes $p>0, q \geq 0$ in the definition of $C_{G S}^{n}(B, B)$, then the resulting cohomology controls the deformations of $B$ to a Drinfel'd (quasi-Hopf) algebra, see GS90b, MS96. Markl has shown in Mar07 that $H_{G S}^{*}(B, B)$ does carries an intrinsic graded bracket. In fact, Markl's construction shows the existence of a bracket for any type of (bi)algebra over an operad or PROP.

For the rest of this section, $B$ will denote either $\mathcal{O}(G)$ or $U \mathfrak{g}$, where $G$ is a reductive algebraic group and $\mathfrak{g}=\operatorname{Lie}(G)$. In these cases, the bialgebra cohomology is easy to compute since $H H^{n}(-, \mathcal{O}(G))=0$ and the $H_{c}^{n}(U \mathfrak{g},-)$ vanish in positive dimensions. Explicitly, if $B=\mathcal{O}(G)$ or $U \mathfrak{g}$ then $H_{G S}^{n}(B, B)=\bigwedge^{n} \mathfrak{g} /\left(\bigwedge^{n} \mathfrak{g}\right)^{\mathfrak{g}}$, where $(\bigwedge \mathfrak{g})^{\mathfrak{g}}$ is the space of $\mathfrak{g}$-invariants in $\bigwedge^{n} \mathfrak{g}$ GS90b. The Schouten bracket on $\bigwedge^{*} \mathfrak{g}$ corresponds to the graded Lie algebra structure on $H_{G S}^{n}(B, B)$. There are no invariants in $\mathfrak{g} \wedge \mathfrak{g}$ and, up to a scalar multiple, there is a unique non-zero invariant in $\bigwedge^{3} \mathfrak{g}$. The infinitesimal bialgebra deformations of $B$ are then elements $r \in \mathfrak{g} \wedge \mathfrak{g}$. The condition $[r, r]=0$ in $H_{G S}^{3}(B, B)$ means either that $r$ is a solution to the classical Yang-Baxter equation (CYBE) (in the case $[r, r]=0$ ) or that it is a solution to the modified CYBE (in the case that $[r, r]$ is a non-zero invariant). Any solution to either of these Yang-Baxter equations gives a Poisson-Lie group structure on $G$. 
The quantization problem for both types of $r$-matrices is solved. For $r$ a solution to the CYBE, the quantization is given by the UDF associated to $r$, see Example 3 of Section 5 and [Dr83]. For the solutions to the modified CYBE, the quantization can be deduced from the "dynamical twist" found in ESS00. The quantizations of [Dr83, and [ESS00, are universal in the sense that they lie in $(U \mathfrak{g} \otimes U \mathfrak{g})[[t]]$, and so they provide a quantum Yang-Baxter matrix in $\operatorname{End}(V \otimes V)[[t]]$ for any representation $V$ of $\mathfrak{g}$. Computing this $R$-matrix from the universal quantization can require great effort. However, in GGS93 a simple explicit "GGS" formula was conjectured to quantize any modified $r$-matrix for $\mathfrak{g}=\mathfrak{s l}(n)$ and $V=k^{n}$, the vector representation. After performing computer checks for over ten thousand cases, the GGS formula was proven correct by Schedler in [Sc00. The proof is far from elementary as it uses intricate combinatorial manipulations to show that the universal solution of [ESS00] coincides with the simple GGS formula. Something is wanting for a simpler proof and real meaning of the GGS formula. It would also be interesting to extend the result to yield elementary quantizations of the modified $r$-matrices in the symplectic and orthogonal cases.

The bialgebra cohomology of $\mathcal{O}(G)$ also guarantees that any deformation is equivalent to one with a deformed product $*$ which is compatible with the original comultiplication $\Delta$. A deformation of the form $(\mathcal{O}(G), *, \Delta)$ is called preferred. Similarly, all bialgebra deformations of $U \mathfrak{g}$ are preferred, although in this case it is the original multiplication which is unchanged. The standard quantization $\mathcal{O}_{q}(G)$ is equivalent to a preferred deformation but no such presentation has been exhibited - even in the simplest case of $\mathcal{O}_{q}(\mathrm{SL}(2))$. As in the case of Lie bialgebra quantization, the difficulty in performing explicit computations seems to be that preferred deformations are linked with a choice of Drinfel'd associator. See BGGS04 for a more complete discussion of deformation quantization as it relates to quantum groups.

Returning to the Yang-Baxter equations, it should be noted that the moduli space of solutions to the MCYBE for a simple Lie algebra has been constructively described by Belavin and Drinfeld in BD82. The solutions fall into a finite disjoint union of components, each of which is determined by an "admissible triple" (certain combinatorial data associated with the root system). In contrast, an explicit classification of solutions to the CYBE is intractable, for it would require as a special case the knowledge of all abelian Lie subalgebras of $\mathfrak{g}$. There is however a non-constructive description of such $r$-matrices in terms of "quasi-Frobenius" Lie algebras, see BD82, Sto91. A Lie algebra $\mathfrak{q}$ is quasi-Frobenius if there is a nondegenerate function $\phi: \mathfrak{q} \wedge \mathfrak{q} \rightarrow k$ which is a two-cocycle in the Chevalley-Eilenberg cohomology. The Lie algebra is Frobenius if the two-cocycle can be taken to be a coboundary, that is, if $\phi(a, b)=F([a, b])$ for some $F \in \mathfrak{q}^{*}$. If $B=\left(B_{i j}\right)$ is the matrix of $\phi$ with respect to some basis $\left\{x_{1}, \ldots, x_{m}\right\}$ of $\mathfrak{q}$, then $r=\sum B_{i j}^{-1} x_{i} \wedge x_{j}$ is a solution to the CYBE. In GG98 it was shown that some solutions to the CYBE arise as degenerations of solutions to the MCYBE, and others do not. Perhaps it may be feasible to describe all of these "boundary" solutions using the Belavin-Drinfel'd triples.

\section{Diagrams of Algebras}

A "diagram" of algebras is a contravariant functor $\mathbb{A}$ from a small category $\mathcal{C}$ to the category of associative $k$-algebras, i.e. a presheaf of algebras over $\mathcal{C}$. So for 
each $i \in \mathrm{Ob}(\mathbb{C})$ there is an algebra $\mathbb{A}^{i}$ and for each morphism $i \rightarrow j$ there is an algebra map $\phi^{i j}: \mathbb{A}^{j} \rightarrow \mathbb{A}^{i}$. Presheaves of algebras are abundant and surface in a variety of contexts: A single algebra is a diagram over a one-object category with only the identity morphism. A diagram over the category with two objects and one non-trivial morphism $u: 0 \rightarrow 1$ is nothing but a homomorphism of algebras $\phi: B \rightarrow A$. The structure sheaf $\mathcal{O}_{X}$ on a quasi-projective variety $X$ is a diagram of commutative algebras over the category $\mathcal{U}$ of open subsets of $X$. Here $\mathcal{U}$ is a category in which the morphisms correspond to inclusion maps.

In a series of papers, Gerstenhaber and Schack developed natural cohomology and deformation theories for diagrams and proved a number of remarkable results. A description of the theory can be found in the survey [GS88. The Hochschild cohomology of sheaves of algebras and abelian categories studied [Hi05] and [LVdb06] are closely related to the Gerstenhaber-Schack diagram cohomology.

Perhaps the most useful and difficult result in diagram cohomology theory is the General Cohomology Comparison Theorem (see Theorem 6) which asserts, in a sense, that the cohomology and deformation theories of an arbitrary diagram are no more general than that of a single algebra. In order to explain more clearly what this means we give a quick review of the basics of the theory.

An $\mathbb{A}$-bimodule $\mathbb{M}$ is a contravariant functor from $\mathbb{C}$ to the category of abelian groups assigning to every $i \in \mathrm{Ob}(\mathcal{C})$ an $\mathbb{A}^{i}$-bimodule $\mathbb{M}^{i}$ and to every morphism $u: i \rightarrow j$ in $\mathcal{C}$ a map $T^{i j}: \mathbb{M}^{j} \rightarrow \mathbb{M}^{i}$ which is required to be an $\mathbb{A}^{j}$-bimodule map. Here, $\mathbb{M}^{i}$ becomes an $\mathbb{A}^{j}$-module by virtue of the algebra homomorphism $\phi^{i j}$.

Just as in the case of a single algebra, there are various descriptions of the diagram cohomology $H_{d}^{*}(\mathbb{A}, \mathbb{M})$. Once the requisite categorical machinery is laid out, one may define $H_{d}^{*}(\mathbb{A}, \mathbb{M})=\operatorname{Ext}_{\mathbb{A}-\mathbb{A}}(\mathbb{A}, \mathbb{M})$. There is also a cochain description which is quite useful and we present this here. There is a cochain complex $\left(C_{d}^{*}(\mathbb{A}, \mathbb{M}), \delta_{d}\right)$ whose homology coincides with $\operatorname{Ext}_{\mathbb{A}-\mathbb{A}}(\mathbb{A}, \mathbb{M})$. The description of $C_{d}^{*}(\mathbb{A}, \mathbb{M})$ has both algebraic and simplicial aspects. The nerve $\Sigma$ of $\mathcal{C}$ is the simplicial complex whose 0 -simplices are the objects of $\mathcal{C}$ and the $p$-simplices are the composable maps $\sigma=\left(i_{0} \rightarrow i_{i} \rightarrow \cdots \rightarrow i_{p}\right)$. For simplicity we write $\sigma=\left(i_{0}, \ldots, i_{p}\right)$. The boundary of $\sigma$ is $\partial \sigma=\sum(-1)^{j} \sigma_{j}$, where $\sigma_{j}$ is the $j$-th face of $\sigma$ obtained by omitting $i_{j}$.

For a diagram $\mathbb{A}$ and $\mathbb{A}$-bimodule $\mathbb{M}$, the $n$-cochains are $C_{d}^{n}(\mathbb{A}, \mathbb{M})=\bigoplus_{p+q=n} C_{d}^{p, q}(\mathbb{A}, \mathbb{M})$, where

$$
C_{d}^{p, q}(\mathbb{A}, \mathbb{M})=\prod_{\substack{p-\text { simplices } \\\left(i_{0}, \ldots, i_{p}\right)}} C^{q}\left(\mathbb{A}\left(i_{p}\right), \mathbb{M}\left(i_{0}\right)\right) .
$$

Fix $\Gamma \in C_{d}^{p, q}(\mathbb{A}, \mathbb{M})$. The diagram coboundary will have two components: $\delta_{\text {alg }} \Gamma \in C_{d}^{p, q+1}(\mathbb{A}, \mathbb{M})$ and $\delta_{\text {simp }} \in C_{d}^{p+1, q}(\mathbb{A}, \mathbb{M})$. The algebraic component is defined by $\left(\delta_{\text {alg }} \Gamma\right)^{\sigma}=\delta_{h}\left(\Gamma^{\sigma}\right)$ where $\delta_{h}: C^{q}\left(\mathbb{A}^{i_{p}}, \mathbb{M}^{i_{0}}\right) \rightarrow C^{q+1}\left(\mathbb{A}^{i_{p}}, \mathbb{M}^{i_{0}}\right)$ is the ordinary Hochschild coboundary operator. The simplicial component is defined as follows. Let $\sigma=\left(i_{0}, \ldots, i_{p+1}\right)$ be a $p+1$-simplex. For faces $\sigma_{j}$ with $1 \leq j \leq p$, we have $\Gamma^{\sigma_{j}} \in C^{q}\left(\mathbb{A}^{i_{p+1}}, \mathbb{M}^{i_{0}}\right)$, while $\Gamma^{\sigma_{0}} \in C^{q}\left(\mathbb{A}^{i_{p+1}}, \mathbb{M}^{i_{1}}\right)$ and $\Gamma^{\sigma_{p+1}} \in C^{q}\left(\mathbb{A}^{i_{p}}, \mathbb{M}^{i_{0}}\right)$. The extreme cases $\Gamma^{\sigma_{0}}$ and $\Gamma^{\sigma_{p+1}}$ lie in different cochain groups than the others, but there are adjustments however which correct this. For $\sigma_{0}$ note that the composite $T^{i_{0} i_{1}} \Gamma^{\sigma_{0}} \in C^{q}\left(\mathbb{A}^{i_{p+1}}, \mathbb{M}^{i_{0}}\right)$. For $\sigma_{p+1}$ define $\Gamma^{\sigma_{p+1}} \phi^{i_{p+1} i_{p}} \in C^{q}\left(\mathbb{A}^{i_{p+1}}, \mathbb{M}^{i_{0}}\right)$ by

$$
\Gamma^{\sigma_{p+1}} \phi^{i_{p+1} i_{p}}\left(a_{1}, \ldots, a_{q}\right)=\Gamma^{\sigma_{p+1}}\left(\phi^{i_{p+1} i_{p}} a_{1}, \ldots, \phi^{i_{p+1} i_{p}} a_{q}\right) .
$$


Now set

$$
\left(\delta_{\text {simp }} \Gamma\right)^{\sigma}=" \Gamma^{\partial \sigma "}=T^{i_{0} i_{1}} \Gamma^{\sigma_{0}}-\Gamma^{\sigma_{1}}+\Gamma^{\sigma_{2}}-\cdots+(-1)^{p} \Gamma^{\sigma_{p}} \phi^{i_{p} i_{p}+1} .
$$

The full diagram coboundary is then

$$
\delta_{d}=\delta_{a l g}+(-1)^{p} \delta_{\text {simp }}
$$

and the diagram cohomology $H_{d}^{*}(\mathbb{A}, \mathbb{M})$ is defined to be the homology of the complex

$$
C_{d}^{*}(\mathbb{A}, \mathbb{M})=\bigoplus_{p+q=n} C_{d}^{p, q}(\mathbb{A}, \mathbb{M}) \quad \text { with } \quad \delta_{d}=\delta_{a l g}+(-1)^{p} \delta_{\text {simp }}
$$

Note that the cohomology of the bottom row $H_{d}^{*, 0}(\mathbb{A}, \mathbb{M})$ coincides with the simplicial cohomology of $\Sigma(\mathbb{C})$ with local coefficients $\mathbb{M}$.

A deformation of $\mathbb{A}$ is a diagram of $k[[t]]$-algebras whose reduction modulo $t$ is $\mathbb{A}$. The diagram cohomology $H_{d}^{*}(\mathbb{A}, \mathbb{A})$ is too large to govern deformations of $\mathbb{A}$ since the simplicial cohomology of $\Sigma(\mathcal{C})$ may not be trivial. There are remedies such as using "asimplicial" cochains or adjoining a terminator to $\mathcal{C}$, see GS88. Naturally, we would like a graded Lie structure on $H_{d}^{*}(\mathbb{A}, \mathbb{A})$ which controls obstructions. It turns out that, unlike the case of a single algebra, the natural bracket on $C_{d}^{*}(\mathbb{A}, \mathbb{A})$ gives the structure of only a homotopy graded Lie algebra. Proving that this bracket descends to a graded Lie structure at the cohomology level would be at best a nasty computation using the cochain description. However, the following very difficult and useful result of GS88 settles this question.

Theorem 6 (General Cohomology Comparison Theorem). Associated to each diagram $\mathbb{A}$ is a single $k$-algebra $\mathbb{A}$ !! such that the cohomology and deformation theories of $\mathbb{A}$ are naturally isomorphic to those of $\mathbb{A} ! !$. In particular, $H_{d}^{*}(\mathbb{A}, \mathbb{A})$ is a Gerstenhaber algebra.

The diagram algebra $\mathbb{A}$ !! is rather complicated and we will not describe it here, although we will see a special case in Section 10. The proof of Theorem 6 relies on the Special Cohomology Comparison Theorem which is the case when $\mathbb{A}$ is a poset. To derive the general case, Gerstenhaber and Schack perform a barycentric subdivision of $\mathbb{A}$. It turns out that the second subdivision of an arbitrary diagram is a poset and subdivision preserves the cohomology. Van den Bergh and Lowen have proved Special Cohomology Comparison Theory for prestacks in LVdb09.

Another important result in diagram cohomology theory is the following theorem which completely reconciles the Kodaira-Spencer manifold deformation theory with the Gerstenhaber-Schack diagram deformation theory.

Theorem 7. Let $X$ be a smooth compact algebraic variety with tangent bundle $T$. Suppose $\mathcal{U}$ be an affine open cover of $X$ and let $\mathbb{A}$ be the restriction of $\mathcal{O}_{X}$ to $\mathcal{U}$. Then there is a Gerstenhaber algebra isomorphism $H_{d}^{*}(\mathbb{A}, \mathbb{A}) \simeq H^{*}\left(X, \bigwedge^{*} T\right)$.

Using the theorem, one sees that

$$
H_{d}^{2}(\mathbb{A}, \mathbb{A}) \simeq H^{2}\left(X, \mathcal{O}_{X}\right) \bigoplus H^{1}(X, T) \bigoplus H^{0}\left(X, \wedge^{2} T\right) .
$$

The middle term consists of the infinitesimal deformations of $X$ in the KodairaSpencer theory. The last term is the space of infinitesimal deformations of $X$ to "non-commutative" spaces; those with vanishing primary obstruction are precisely the Poisson structures on $X$ and, by Theorem 1 , these are quantizable. The meaning of the first term of $H_{d}^{2}(\mathbb{A}, \mathbb{A})$ is not well-understood. 
Besides applications to geometric situations, diagrams naturally arise in other contexts. For example, given an algebra $A$ and an $A$-module $M$, one can deform the action of $A$ on $M$ in the evident way, and it is relatively easy in this case to deduce the appropriate deformation cohomology. More generally, one can simultaneously deform $A$ and its action on $M$ in a compatible way. These situations are special cases of diagram deformations. Indeed, the original $A$-module structure on $M$ is simply an algebra homomorphism $\phi: A \rightarrow \operatorname{End}(M)$, and hence is a diagram. Deformations of this diagram yield the various possibilities of deforming $A$, the action of $A$ on $M$, or both. The general theory automatically yields appropriate cohomology and obstruction theories. In Section 10 the diagram cohomology theory will be used to cohomologically explain how certain rigid algebras can appear in naturally parametrized families.

\section{Deforming RELATions}

Suppose an algebra is given as $A=T X / J$ where $X$ is the $k$-module spanned by finitely many generators $x_{i}, T X$ is the tensor algebra, and $J$ is the ideal of relations. If $J_{t}$ is an ideal of $T X[[t]]$ which reduces to $J$ modulo $t$, then a natural question is whether $A_{t}=T X[[t]] / J_{t}$ is a deformation of $A$ or not. Associativity of $A_{t}$ is automatic but to be a deformation it must be flat as a $k[[t]]$-module. There is no efficient way in general to determine if the relations in $J_{t}$ insure flatness. An elementary case where flatness fails is the following: Let $A=k[x, y, z]$ and let $J_{t}$ be generated by $y x-(1+t) t x y, z x-x z-t y^{2}$ and $y z-z y$. When $t=0$ all variables commute and the polynomial algebra $k[x, y, z]$ is obtained. For $t \neq 0$, the deformed relations allow for a $\mathrm{PBW}$-type ordering in which every monomial of $A_{t}$ can be reduced to one of the form $x^{i} y^{k} z^{k}$. However, the element $t(1+t) y^{3}$ lies in $J_{t}$ and so $A_{t}$ has $t$-torsion and thus is not flat.

Flatness is relatively easy to check for certain deformations of Koszul algebras, which comprise an important class of quadratic algebras. An algebra $A$ is quadratic if $A=T X / J$, with $J$ is generated by relations $R \subset X \otimes X$. Since the relations are homogeneous, such algebras are $\mathbb{N}$-graded, $A=\bigoplus_{i \geq 0} A[i]$ and $\operatorname{dim} A[i]<\infty$ for each $i$. In particular, $A[0]=k$. A quadratic algebra $A$ is Koszul if its dual $A^{!}$is isomorphic to the Yoneda algebra $\operatorname{Ext}_{A}^{*}(k, k)$. Variations of the following fundamental theorem have appeared in several places in the literature, most notably in the works of Drinfel'd Dr86] and Braverman-Gaitsgory [BG96].

Theorem 8 (Koszul Deformation Criterion). Suppose that $A=T X / J$ is Koszul and $A_{t}=T X[[t]] / J_{t}$, where $J_{t}$ is generated by relations $R_{t} \subset(X \otimes X)[[t]]$ which reduce to $J$ modulo $t$. Then $A_{t}$ is a deformation of $A$ if and only if $A_{t}[3]$ is a flat $k[[t]]-$ module.

The point of the theorem is that in the Koszul case, flatness in dimensions greater than 3 is a consequence of flatness in degree 3. Flatness in the cases of degrees 1 and 2 is automatic.

One of the most interesting and explicit uses of the Koszul deformation criterion has been carried out by Etingof and Ginzburg in the theory of symplectic reflection algebras, which are deformations of crossed product algebras $S V \ltimes G$, see [EG02]. One can try to deform $S V \ltimes G$ by imposing additional relations of the form $x y-y x=$ 
$\kappa(x, y)$ where $x, y \in V$ and $\kappa(x, y)=-\kappa(y, x) \in \mathbb{C} G$. For an arbitrary skewsymmetric function $\kappa$, the underlying vector space of the resulting algebra, $A_{\kappa}$, will be smaller than that of $A_{0}=S V \ltimes G$ - that is, the deformation will not be flat.

In the case where $V$ is a symplectic vector space and $G \in S p(V)$, Etingof and Ginzburg have an explicit and remarkable classification of which skew forms $\kappa$ lead to deformations. To describe these, we first need some notation. Suppose $V$ is a complex vector space equipped with a skew bilinear form $\omega: V \times V \rightarrow \mathbb{C}$, and let $G$ be a finite subgroup of $S p(V)$. An element $s \in G$ is a symplectic reflection if the rank of $1-s$ is 2 . The set of all symplectic reflections is denoted $S$. For each $s \in S$, let $\omega_{s}$ denote the form on $V$ with radical $\operatorname{Ker}(1-s)$ and which coincides with $\omega$ on $\operatorname{Im}(1-s)$. The triple $(V, \omega, G)$ is indecomposable if $V$ can not be split into a non-trivial direct sum of $G$-invariant symplectic subspaces.

Theorem 9 (Etingof-Ginzburg). Suppose $(V, \omega, G)$ is an indecomposable triple, and let $\kappa: V \times V \rightarrow \mathbb{C} G$ be a skew form. Then $A_{\kappa}$ is a flat deformation of $S V \ltimes G$ if and only if there exists a $G$-invariant function $c: S \rightarrow \mathbb{C}, s \mapsto c_{s}$ and a constant $t$, such that

$$
\kappa(x, y)=t \omega(x, y)+\sum_{s \in S} c_{s} \omega_{s}(x, y) s .
$$

As stated earlier, the applications of symplectic reflection algebras are many. Here is one particularly interesting one. The center of $S V \ltimes G$ is the algebra $(S V)^{G}$ of $G$-invariant polynomial functions, which can be viewed as the functions on the orbit space $V / G$. If $e=\frac{1}{|G|} \sum_{g \in G} g$ is the symmetrizing idempotent in $\mathbb{C} G$, then the spherical subalgebra of $A_{\kappa}$ is defined to be $e A_{\kappa} e$. It is known that $e A_{0} e \simeq(S V)^{G}$, and so $e A_{\kappa} e$ provides a non-commutative deformation of $(S V)^{G}$. However, if $t=0$ then the algebra $e A_{\kappa} e$ is commutative. Thus the symplectic reflection algebras can provide geometric deformations of $V / G$.

Returning to Theorem 8 , there are algebras where, unlike the symplectic reflection algebras, there is no evident ordered or PBW-type basis of $A_{t}$. For example, Sklyanin (or elliptic) deformations of polynomial algebras have this property. The simplest case is the algebra with generators $\{x, y, z\}$ and relations

$$
a x^{2}+b y z+c z y=0, \quad a y^{2}+b z x+c x z=0, \quad a z^{2}+b x y+c y x=0 .
$$

The triple $(a, b, c)=(0,1,-1)$ gives the polynomial algebra $k[x, y, z]$, but for generic $(a, b, c)$ the relations are such that there is no PBW-type basis. One way to prove flatness is to associate certain geometric data (an elliptic curve $\mathcal{E}$ and point $\eta \in \mathcal{E}$ ) to the algebra in question. The geometric information allows one to construct a factor ring of the Sklyanin algebra which can be exploited to establish flatness. A survey of elliptic deformations of polynomial algebras can be found in Od02.

\section{VARIATION OF ALGEBRAS}

As mentioned in section 4, an algebra with $H^{2}(A, A)=0$ may depend essentially on parameters and so the classic deformation theory of $A$ does not detect this dependence. If we instead pass to an appropriate diagram of algebras, it is possible in many cases to detect the dependence of $A$ on parameters from the diagram cohomology and construct the new algebra with the concept of algebra variation. In this section we give a brief account of [GG08b].

Suppose that we have $k$-algebras $A, B, B^{\prime}$ and monomorphisms $\phi: B \rightarrow A$ and $\phi^{\prime}: B^{\prime} \rightarrow A$ such that $A$ is generated by the images $\phi(B)$ and $\phi^{\prime}\left(B^{\prime}\right)$. If $V$ is the 
direct sum of the underlying $k$-modules of $B$ and $B^{\prime}$ then $A=T V / J$, where $J$ is the ideal of $T V$ generated by relations which we write in the form $R\left(\phi(b), \phi\left(b^{\prime}\right)\right)$ for $b \in B$ and $b^{\prime} \in B^{\prime}$. In this case we have a diagram $\mathbb{A}$ over the poset $\mathcal{C}=\left\{0,1,1^{\prime}\right\}$ :

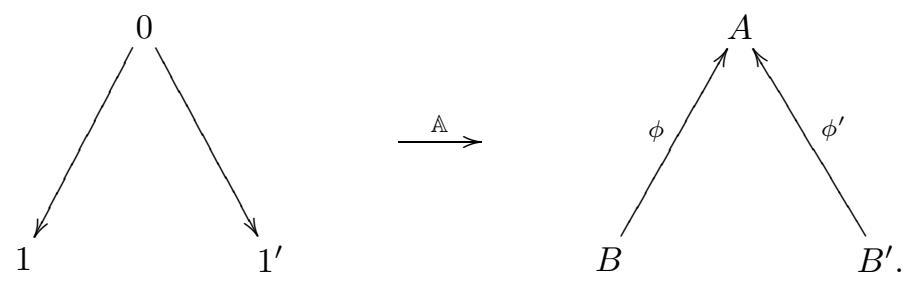

Now consider a deformation $\mathbb{A}_{t}$ of $\mathbb{A}$ in which the algebras $A, B$, and $B^{\prime}$ remain fixed but the homomorphism $\phi$ is deformed as $\phi_{t}=\phi+t \phi_{1}+t^{2} \phi_{2}+\cdots$ and similarly assume $\phi^{\prime}$ is deformed to $\phi_{t}^{\prime}$. We can use the same relations determining $A$ with deformed inputs to construct a new algebra $A_{t}$.

Definition 3. Suppose $A, B, B^{\prime}, V, R, \mathbb{A}$ and $\mathbb{A}_{t}$ are as above. Let $J_{t}$ be the ideal of $T V[[t]]$ generated by all elements of the form $R\left(\phi_{t}(b), \phi_{t}^{\prime}\left(b^{\prime}\right)\right)$ for $b \in B$ and $b^{\prime} \in B^{\prime}$. The algebra $A_{t}=T V[[t]] / J_{t}$ is called a variation of $A$.

A variation $A_{t}$ is certainly associative but there is no guarantee that it is flat, and as noted earlier, there is in general no easy way to determine when such algebras are flat. The concept of variation can clearly be generalized by letting $A$ be generated by more than two subalgebras.

It is important to note that not all algebras of the form $T V[[t]] / J_{t}$ where $J_{t}$ is an ideal of $T V[[t]]$ with $J_{0}=J$ are variations of $A$. As an example, take $A$ to be commutative. Then we have in $J$ all relations of the form $\phi(b) \phi^{\prime}\left(b^{\prime}\right)-\phi^{\prime}\left(b^{\prime}\right) \phi(b)$. The ideal $J_{t}$ defining the variation $A_{t}$ will therefore have all relations of the form $\phi_{t}(b) \phi_{t}^{\prime}\left(b^{\prime}\right)-\phi_{t}^{\prime}\left(b^{\prime}\right) \phi_{t}(b)$ and so $A_{t}$ remains commutative.

Let us return now to the deformation of the diagram $\mathbb{A}$ in the above figure obtained by replacing $\phi$ with $\phi_{t}$ and $\phi^{\prime}$ with $\phi_{t}^{\prime}$. Its infinitesimal lies in $H_{d}^{2}(\mathbb{A}, \mathbb{A})$ and is the class of a cocycle of the form $\Gamma=\left(\Gamma_{A}, \Gamma_{B}, \Gamma_{B^{\prime}}, \Gamma_{B A}, \Gamma_{B^{\prime} A}\right)$ with

$$
\begin{aligned}
\Gamma_{A} \in H H^{2}(A, A), \quad \Gamma_{B} \in H H^{2}(B, B), \quad \Gamma_{B^{\prime}} \in H H^{2}\left(B^{\prime}, B^{\prime}\right), \\
\Gamma_{B A} \in H H^{1}(B, A) \quad \text { and } \quad \Gamma_{B^{\prime} A} \in H H^{1}\left(B^{\prime}, A\right) .
\end{aligned}
$$

The first three components of $\Gamma$ have algebraic dimension 2 and simplicial dimension 0 while the last two have algebraic and simplicial dimension 1 as these correspond to the 1 -simplices of the underlying category. The deformation $\mathbb{A}_{t}$ may be viewed as an integral of this cohomology class. We also assign this class to the variation $A_{t}$.

Even if the algebras $A, B$, and $B^{\prime}$ are absolutely rigid, $H_{d}^{2}(\mathbb{A}, \mathbb{A})$ may not vanish in general as $H H^{1}(B, A)=\operatorname{Der}(B, A) \neq 0$ and similarly for $H H^{1}\left(B^{\prime}, A\right)$. In this case, $\Gamma$ obviously can be taken to be of the form $\left(0,0,0, \Gamma_{B A}, \Gamma_{B^{\prime} A}\right)$. However, if the characteristic of $k$ is zero, then we may further assume that $\Gamma=\left(0,0,0,0, \Gamma_{B^{\prime} A}\right)$. 
Remark 1. The diagram algebra $\mathbb{A} ! !$ associated to $\mathbb{A}$ (see Theorem $[$ ) can be viewed as the algebra of $3 \times 3$ matrices of the form

$$
\left[\begin{array}{ccc}
a_{1} & a_{2} & a_{3} \\
0 & b & 0 \\
0 & 0 & b^{\prime}
\end{array}\right], \quad \text { with } a_{i} \in A, b \in B, b^{\prime} \in B^{\prime}
$$

where the multiplication in $\mathbb{A} ! !$ uses the convention that $b a=\phi(b) a$ and similarly for $b^{\prime} a$. Even in this simple case it is difficult to see how to canonically relate the cohomology and deformations of $\mathbb{A}$ with those of $\mathbb{A} ! !$.

We end with a reconsideration of the first quantized Weyl algebra $A_{q}=\mathbb{C}[x, y]$ with relation $q x y-y x=1$. Having already remarked that $A_{q}$ is not isomorphic to $A_{1}$ for $q$ near 1 , yet $H H^{2}\left(A_{1}, A_{1}\right)=0$. Consider now whether $A_{1}$ can be varied to $A_{q}$. Using our earlier notation, suppose $A=A_{1}, B=\mathbb{C}[x]$, and $B^{\prime}=\mathbb{C}[y]$ and let $\phi: B \rightarrow A$ and $\phi: B^{\prime} \rightarrow A$ be the inclusion maps. All of these algebras are absolutely rigid. Thus, based on the comments above, it suffices to vary the inclusion morphism of $\mathbb{C}[y]$ into $A_{1}$. The question becomes whether there exists an element $y^{\prime} \in A_{1}[[t]]$ of the form $y+t \eta_{1}+t^{2} \eta_{2}+\cdots, \eta_{i} \in A_{1}$ such that the relation $\left[x, y^{\prime}\right]=x y^{\prime}-y^{\prime} x=1$ is equivalent to having $[x, y]=1-t x y$, for this would give $(1+t) x y-y x=1$, i.e., $A_{q}[[t]]$ with $q=1+t$. There are indeed elements $y^{\prime}$ of the desired form. In GG08b, it is shown that one may take

$$
y^{\prime}=y+a_{1}(t) x y^{2}+a_{2}(t) x^{2} y^{3}+\ldots, \quad \text { where } a_{r}(t)=\frac{t^{r+1}}{(1+t)^{r+1}-1} .
$$

Thus, $A_{q}$ is a variation of $A_{1}$. It is easy to use the formula for $y^{\prime}$ to show that the corresponding diagram infinitesimal is $\Gamma=(0,0,0,0, \delta)$, where $\delta \in \operatorname{Der}\left(\mathbb{C}[y], A_{1}\right)$ is the derivation with $\delta(y)=x y^{2}$. This is a non-trivial cohomology class in $H_{d}^{2}(\mathbb{A}, \mathbb{A})$ and so the diagram cohomology has detected the variation from $A_{1}$ to $A_{q}$.

It is instructive to note that in the power series representation of the $a_{r}(t)$ there could be no value of $t$ for which all the series converge, for each $a_{r}(t)$ is a rational function with a pole wherever $t$ has the form $\omega-1$ where $\omega$ is an $(r+1)$ st root of unity and every neighborhood of 0 in $\mathbb{C}$ contains infinitely many of these. Those $A_{q}$ with $q$ a root of unity are in some sense 'unreachable' from $A_{1}$. Nevertheless, $y^{\prime}$ can actually be evaluated for any complex number $t$ with $1+t$ not a root of unity.

\section{REFERENCES}

[Ba68] Barr M.: Harrison homology, Hochschild homology, and triples, J. Alg. 8, (1968), 314-323.

[BFFLS78] Bayen F., Flato M., Fronsdal C., Lichnerowicz A., Sternheimer D.: Deformation theory and quantization I: Deformation of symplectic structures; II: Physical applications, Ann. Phys. (NY) 111 (1978), 61-110; 111-151.

[BD82] Belavin A. and Drinfel'd V., Solutions of the classical Yang-Baxter equation for simple Lie algebras. (Russian), Funktsional. Anal. i Prilozhen. 16 (1982) no. 3, 1-29.

[BB92] F. Bergeron and N. Bergeron.: Orthogonal idempotents in the descent algebra of type $B_{n}$ and applications, J. Pure Appl. Alg., 79(2) (1992), 109-129.

[BW95] Bergeron N. and Wolfgang H.L.: The decomposition of Hochschild cohomology and Gerstenhaber operations, J. Pure Appl. Algebra, 104(3) (1995), 243-265.

[BTY07] Bieliavsky, P., Tang, X., and Yao, Y.: Rankin-Cohen brackets and formal quantization. Adv. Math. 212 (2007), no. 1, 293-314. 
[BGGS04] Bonneau P., Gerstenhaber M., Giaquinto A., Sternheimer D.: Quantum groups and deformation quantization: explicit approaches and implicit aspects. J. Math. Phys. 45(10) (2004), 3703-3741.

[BG96] Braverman A. and Gaitsgory D.: Poincare-Birkhoff-Witt theorem for quadratic algebras of Koszul type. J. Algebra 181 (1996), no. 2, 315-328.

[CGW04] Caldararu A., Giaquinto A., and Witherspoon S.: Algebraic deformations arising from orbifolds with discrete torsion, J. Pure Appl. Alg., 187(1) (2004), 51-70.

[CF00] Cattaneo A.S. and Felder G.: A path integral approach to the Kontsevich quantization formula, Comm. Math. Phys., 212 (3) (2000) 591-611.

[CKTB05] Cattaneo A., Keller B., Torossian C., and Bruguieres A.: Déformation, quantification, théorie de Lie. (French) [Deformation, quantization, Lie theory] Panoramas et Synthèses [Panoramas and Syntheses], 20. Société Mathématique de France, Paris, 2005.

[CM04] Connes A. and Moscovici H.: Rankin-Cohen brackets and the Hopf algebra of transverse geometry, Mosc. Math. J. 4 (2004), no. 1, 111-130.

[Do60] Douady A.: Obstruction primaire á la déformation, Séminaire Henri Cartan, 4, 196061, exposé.

[DMZ07] Doubek, M., Markl, M., and Zima, P.: Deformation theory (lecture notes), Arch. Math. (Brno) 43 (2007), no. 5, 333-371.

[Dr83] Drinfel'd V. G.: On constant, quasiclassical solutions of the classical Yang-Baxter equation, Soviet Math. Dokl., 28(3), 667-671.

[Dr86] Drinfel'd V. G.: On quadratic commutation relations in the quasiclassical case [translation of Mathematical physics, functional analysis (Russian), 25-34, 143, "Naukova Dumka", Kiev, 1986; MR0906075 (89c:58048)]. Selected translations. Selecta Math. Soviet. 11 (1992), no. 4, 317-326.

[Dr92] Drinfe'd V.G.: On some unsolved problems in quantum group theory, Lect. Notes Math., 1510 (1992), 1-8.

[EG02] Etingof P. and Ginzburg V.: Symplectic reflection algebras, Calogero-Moser space, and deformed Harish-Chandra homomorphism, Invent. Math., 147 (2002), no. 2, 243-348.

[EK96] Etingof P. and Kazhdan D.: Quantization of Lie bialgebras. I., Selecta Math. (N.S.) 2 (1996), no. 1, 1-41.

[ESS00] Etingof P., Schedler T. and Schiffman O.: Explicit quantization of dynamical $r$ matrices for finite dimensional semisimple Lie algebras, J. Amer. Math. Soc. 13(3) (2000), 595-609.

[FN57] Frölicher A. and Nijenhuis A.: A theorem on stability of complex structures. Proc. Nat. Acad. Sci. 43 (1957), 239-241.

[Fr07] Fronsdal C.: Quantization on curves, Lett. Math. Phys. 79 (2007), no. 2, 109-129. (appendix by Kontsevich M.)

[Ga90] Garsia A.: Combinatorics of the free Lie algebra and the symmetric group, Analysis, et Cetera, 309-382, Academic Press, Boston, MA, 1990.

[Ge63] Gerstenhaber M.: The cohomology structure of an associative ring, Ann. Math. 78 (1963), 267-288.

[Ge64] Gerstenhaber M.: On the deformation of rings and algebras, Ann. Math. (2) 79 (1964), 59-103.

[Ge68] Gerstenhaber M.: On the deformation of rings and algebras III, Ann. Math. (2) 88(1), 1-34.

[GG98] Gerstenhaber M. and Giaquinto A,: Boundary solutions of the quantum Yang-Baxter equation and solutions in three dimensions, Lett. Math. Phys. 44 (1998), no. 2, 131141.

[GG08a] Gerstenhaber M. and Giaquinto A.: Graphs, Frobenius functionals, and the classical Yang-Baxter equation, arXiv:0808.2423v1.

[GG08b] Gerstenhaber M. and Giaquinto A.: Variation of Algebras, preprint, 2008.

[GGS93] Gerstenhaber M., Giaquinto A. and Schack S.D.: Construction of quantum groups from Belavin-Drinfel'd infinitesimals, Quantum deformations of algebras and their representations, Israel Math. Conf. Proc. 7, 45-64, Bar-Ilan Univ., Ramat Gan, 1993.

[GS86] Gerstenhaber, M. and Schack, S. D.: Relative Hochschild cohomology, rigid algebras and the Bockstein, J. Pure Appl. Alg. 43 (1986), 53-74. 
[GS87] Gerstenhaber, M. and Schack, S. D.: A Hodge-type decomposition for commutative algebra cohomology, J. Pure Appl. Alg. 48 (1987), 229-247.

[GS88] Gerstenhaber M., Schack S.D.: Algebraic cohomology and deformation theory. Deformation theory of algebras and structures and applications (Il Ciocco, 1986), 11-264, NATO Adv. Sci. Inst. Ser. C Math. Phys. Sci. 247, Kluwer Acad. Publ. Dordrecht 1988.

[GS90a] Gerstenhaber M., Schack S.D.: Bialgebra cohomology, deformations, and quantum groups, Proc. Nat. Acad. Sci, 87(1) (1990), 478-481.

[GS90b] Gerstenhaber M., Schack S.D.: Algebras, bialgebras, quantum groups, and algebraic deformations, Deformation theory and quantum groups with applications to mathematical physics (Amherst, MA, 1990), 51-92, Contemp. Math., 134, Amer. Math. Soc., Providence, RI, 1992.

[GZ98] Giaquinto A. and Zhang J.: Bialgebra actions, twists, and universal deformation formulas, Jour. Pure Appl. Alg. 128(02) (1998), 133-151.

[Hi05] Hinich V.: Deformations of sheaves of algebras, Adv. Math. 195(1) (2005), 102-164 (math.AG/0310116).

[KS58] Kodaira K. and Spencer D.C.: On deformations of complex analytic structures, Ann. Math. (2) 67 (1958), 328-466.

[Ko97] Kontsevich M.: Deformation quantization of Poisson manifolds, Lett. Math. Phys, 66(3) (2003), 157-216 (q-alg/9709040).

[Ko01] Kontsevich M.: Deformation quantization of algebraic varieties, EuroConférence Moshé Flato 2000, Part III (Dijon), Lett. Math. Phys. 56(3) (2001), 271-294.

[KLO01] Kulish P. P., Lyakhovsky V.D. and del Olmo M. A.: Chains of twists for classical Lie algebras, J. Phys. A: Math. Gen. 32 (1999), 8671-8684.

[LVdb06] Lowen W. and Van den Bergh M.: Deformation theory of abelian categories, Trans. Amer. Math. Soc. 358 (2006), 5441-5483.

[LVdb09] Lowen W. and Van den Bergh M.: A Hochschild cohomology Comparison Theorem for prestacks, Trans. Amer. Math. Soc., to appear.

[LS02] Lyakhovsky V. D. and Samsonov M. E.: Elementary parabolic twist, J. Algebra Appl. 1 (2002), no. 4, 413-424.

[Lo88] Loday J-L.: Partition eulérienne et opérations en homologie cyclique, C. R. Acad. Sci. Paris Sér. I Math. 307(7) (1988), 283-286.

[Lo89] Loday J-L.: Opérations sur l'homologie cyclique des algèbres commutatives, Invent. Math. 96(1) (1989), 205-230.

[Mat97] Mathieu O.: Homologies associated with Poisson structures, Deformation theory and symplectic geometry (Ascona, 1996), Math. Phys. Stud., 20, Kluwer Acad. Publ., Dordrecht, (1997), 177-199.

[Mar07] Markl M.: Intrinsic brackets and the $L_{\infty}$-deformation theory of bialgebras, arXiv:math/0411456v6

[MS96] Markl M. and Shnider S.: Cohomology of Drinfeld algebras: a homological algebra approach, Internat. Math. Res. Notices (9) (1996), 431-445.

[Od02] Odesskii, A. V., Elliptic algebras. (Russian) Uspekhi Mat. Nauk 57 (2002), no. 6(348), 87-122; translation in Russian Math. Surveys 57 (2002), no. 6, 1127-1162.

[Pi06] Pinczon G.: On two theorems about symplectic reflection algebras, math.QA/0612690.

[Ri67] Richardson R.W.: On the rigidity of semi-direct products of Lie algebras, Pacific J. Math., 22(2) (1967), 339-344.

[Ri57] Riemann B.: Theorie der Abel'schen Functionen, Journal für die reine und angewandte Mathematik, 54 (1857), 101-155)

[Sc00] Schedler T., Proof of the GGS conjecture. Math. Res. Lett. 7 (2000), no. 5-6, 801-826.

[Ste] Sternheimer D.: The deformation philosophy, quantization and noncommutative space-time structures, this volume.

[Sta63] Stasheff J.,: Homotopy associativity of $H$-spaces. I, II. Trans. Amer. Math. Soc. 108 (1963), 275-292;

[Sta93] Stasheff J.: The intrinsic bracket on the deformation complex of an associative algebra, J. Pure Appl. Algebra 89 (1993), 231235.

[Sto91] Stolin A., On rational solutions of Yang-Baxter equation for $\mathfrak{s l}(n)$, Math. Scand. 69 (1991), 57-80. 
[VdB07] Van den Bergh M.: On global deformation quantization in the algebraic case, J. Algebra 315(1) (2007), 326-395 (math. AG/0603200).

[Ye05] Yekutieli A.: Deformation quantization in algebraic geometry, Adv. Math. 198(1) (2005), 383-432.

Mathematics and Statistics, Loyola University Chicago, Chicago IL 60626 tonyg@math.luc.edu 